\title{
Biosynthesis of Natural and Hyperelongated Chondroitin Sulfate Glycosaminoglycans: New Insights into an Elusive Process
}

\author{
Peter J. Little*,a,b,c Mandy L. Ballinger ${ }^{\mathrm{a}}$, Micah L. Burch ${ }^{\mathrm{a}, \mathrm{c}}$ and Narin Osman ${ }^{\mathrm{a}, \mathrm{b}}$

\begin{abstract}
${ }^{a}$ Diabetes and Cell Biology Laboratory, Vascular and Hypertension Division, BakerIDI Heart and Diabetes Institute, Melbourne, VIC, Australia 3004 and ${ }^{b}$ Medicine and ${ }^{c}$ Immunology Departments, Central and Eastern Clinical School, Alfred Hospital, Monash University, Melbourne, VIC, 3004, Australia
\end{abstract}

\begin{abstract}
Proteoglycans are important components of the extracellular matrix of all tissues. Proteoglycans are comprised of a core protein and one or more covalently attached glycosaminoglycan (GAG) chains. The major chondroitin sulfate (CS) and dermatan sulfate (DS) proteoglycans are aggrecan, versican, biglycan and decorin. Cells synthesize GAGs of natural or basal lengths and the GAG chains are subject to considerable growth factor, hormonal and metabolic regulation to yield longer GAG chains with altered structure and function. The mechanism by which the CS/DS GAG chains are polymerized is unknown. Recent work has identified several monosaccharide transferases which when co-expressed yield GAG polymers and the length of the polymers depends upon the pair of enzymes coexpressed. The further extension of these chains is regulated by signaling pathways. Inhibition of these latter pathways may be a therapeutic target to prevent the elongation which is associated with increased binding of atherogenic lipids and the disease process of atherosclerosis.
\end{abstract}

Keywords: Proteoglycans, chondroitin sulfate, glycosaminoglycans, enzymology, polymerases, signaling.

\section{INTRODUCTION}

Proteoglycans are an integral part of the extracellular matrix of all tissues [1]. In some tissues proteoglycans are a low component by mass but are highly important because they are both structural molecules and actively involved in critical cell functions including proliferation, migration, phenotypic expression and secretion [2]. Proteoglycans are complex macromolecules comprised of a core protein and one or more covalently attached glycosaminoglycans (GAG) chains so that structurally they are both proteins and carbohydrates. The spectrum of properties of proteoglycans is determined by both the core proteins and the GAG chains. Core proteins determine the cellular location and serve as scaffolds for the GAG chains and the highly ionic chains determine physical properties of tissues and bind factors including growth factors and apolipoproteins. The complementary roles of core protein and GAG are exemplified by the observation that binding of Low Density Lipoproteins (LDL) to GAG chains of intact proteoglycans occurs with an order of magnitude higher affinity than the binding to free GAG chains [3]. There is considerable interest in the biosynthetic pathways determining proteoglycan and particularly GAG structure because GAG chains are implicated in many fundamental biological and pathological processes [4]. The signaling pathways regulating core protein synthesis and GAG elongation are of specific interest. The pathways regulating these two processes are distinct with initial evidence indicating that the signaling pathway regulating core protein expression is aligned to that of the cell cycle whereas the

*Address correspondence to this author at the Head, Diabetes and Cell Biology Laboratory, BakerIDI, Heart and Diabetes Institute, St. Kilda Rd Central, PO Box 6492, Melbourne, VIC 8008, 75 Commercial Road, Melbourne, VIC 3004, Australia; Tel: +61 38532 1203; Fax: +61 38532 1100; E-mail: peter.little@bakeridi.edu.au signaling pathway regulating elongation of GAGs has some distinct properties [5]. The biochemical mechanisms and signaling pathways regulating the synthesis of GAG chains are the focus of this review.

The GAG chains consisting of a tetrasaccharide linkage region and repeating disaccharide moieties are sulfated galactosaminoglycans in the case of chondroitin sulfate (CS) and dermatan sulfate (DS) proteoglycans. CS and DS GAGs are initially synthesized as CS GAGs (containing glucuronic acid (GlcA)) and the activity of the enzyme C5-epimerase to isomerize GlcA to Iduronic acid (IdoA) by definition generates DS GAGs [6]. Thus this review refers to CS GAG synthesis but the discussion, allowing for the action of C5-epimerase, also relates to DS GAG chains. The major CS proteoglycans are aggrecan, which forms a substantial structural component of cartilage and is composed of both CS and keratan sulfate GAG chains, and the large CS proteoglycan versican which is prominent in blood vessels. The smaller leucine-rich proteoglycans, biglycan and decorin, are CS GAG with some epimerase modification rendering them DS proteoglycans and they are also prominently expressed in blood vessels [7]. The highly ionized state of the sulfated GAG chains leads to their role in determining the physical properties of tissues such as the resistance to compression of cartilage provided by aggrecan. The loss of CS from cartilage is a major cause of osteoarthritis [8]. Conversely, the accumulation of biglycan in the wall of injured blood vessels leads to the trapping of apolipoproteins and is recognized as the initiating factor in atherosclerosis which underlies most cardiovascular disease $[9,10]$.

Although GAGs have critical biological roles the precise mechanism by which CS GAG polymers are synthesized is unknown. Until recently, no in vitro system has proven capable of synthesizing GAG chains of similar length to those 
secreted by cells and occurring in tissues. GAG chains do not have a discrete molecular weight but rather have a spectrum of values which can be readily observed by SDS-PAGE (see Fig. (1) Lower Panel) or size exclusion analysis plots [11]. The range of normal GAG chain lengths is very wide and extends from 20 to approximately $60 \mathrm{KDa}$ corresponding to approximately 40-120 disaccharides. Proteoglycans, such as biglycan, have endogenous synthesis and superimposed upon it the phenomena of GAG hyperelongation. In this response, agonists, such as hormones and growth factors stimulate signaling pathways leading to elongation and thus an increase in the average molecular weight $[12,13]$ (see Fig. 1). Growth factor signaling pathways can also alter epimerization and the disaccharide sulfation pattern and all these structural alterations are considered to have functional consequences [14].

This article addresses the question of whether the hyperelongation effect is an extension of or is distinct from the basic GAG chain polymerizing process. To relate structure to function we use the example of the binding of CS/DS GAG chains to apolipoproteins on lipids and the GAG structural features affecting this interaction [15]. We further consider if it may be possible to dissect the basic mechanisms of GAG synthesis associated with the physiological roles of these molecules, from the mechanisms of hyperelongation that represent a target for the prevention of lipid retention diseases such as atherosclerosis and calcific aortic valve disease (CAVD) [14, 16, 17].

\section{BIOCHEMISTRY OF GAG SYNTHESIS}

CS/DS GAGs on proteoglycans are synthesized by the initial addition of a xylose (Xyl) residue to a specific serine in the core protein followed by the sequential addition of two galactose (Gal) and one GlcA residues to form the tetrasaccharide linkage region (GlcA $\beta 1-3 \mathrm{Gal} \beta 1-3 \mathrm{Gal} \beta 1-4 \mathrm{Xyl} \beta 1-\mathrm{O}-$ Ser) [18] (see Fig. 1). The Xyl and Gal residues in this region can be modified by phosphorylation and sulfation and these modifications have a profound influence on subsequent GAG chain synthesis [19]. Transfer of a $\beta$ GalNAc residue to the terminal GlcA of the linkage region initiates chain po-

\section{Core Protein Glycosaminoglycan chain}
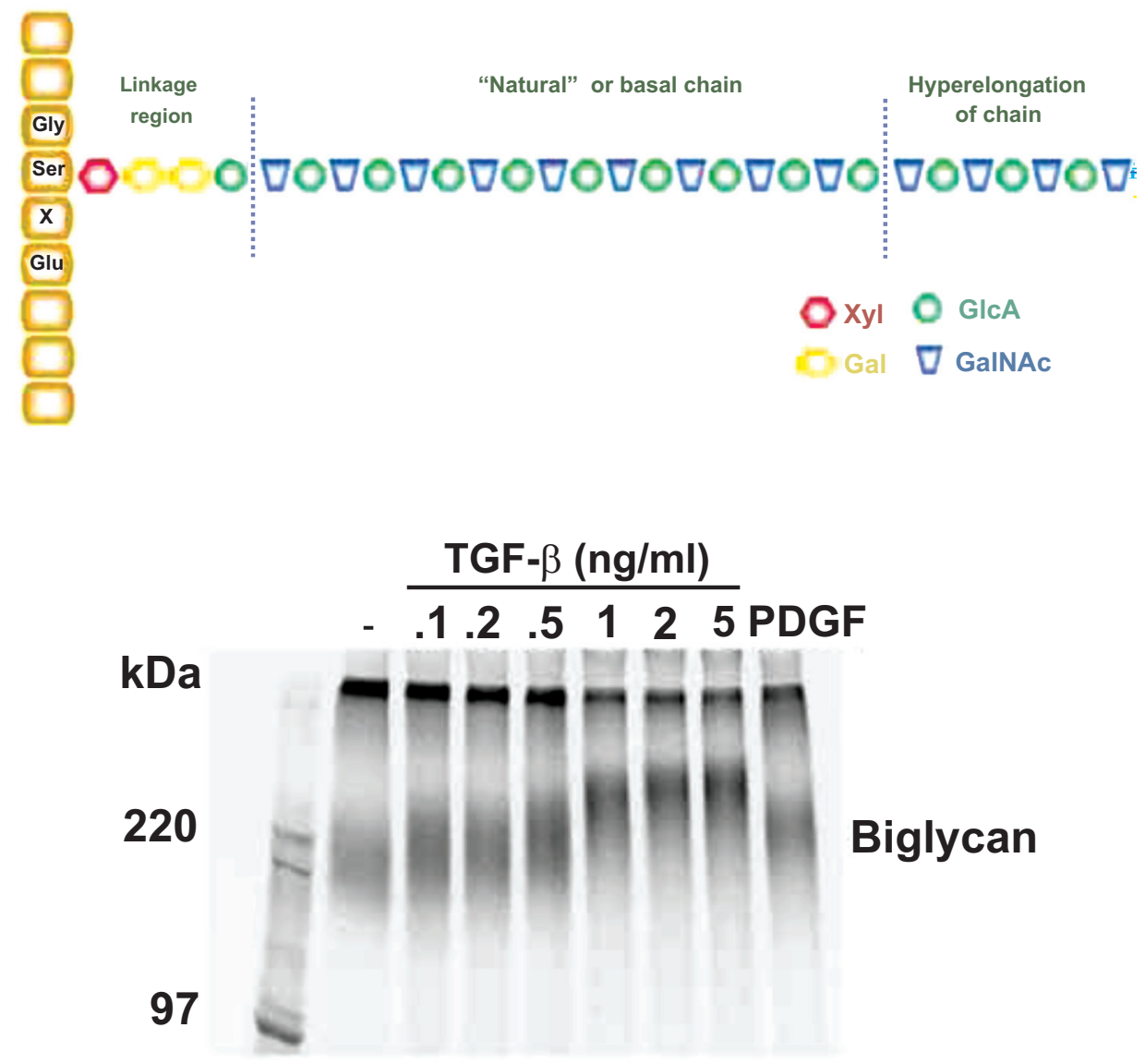

Fig. (1). Schema and actual experimental depiction of the structure of "natural" and "hyperelongated" chondroitin and dermatan sulfate glycosaminoglycan (GAG) chains. Natural or basal GAG chains are defined in the text and the hyperelongation of GAG chains (as indicated in the figure - Upper Panel) occurs, for example, when vascular smooth muscle cells are stimulated with growth factors such as TGF $\beta$. The electrophoretic mobility of intact ${ }^{35} \mathrm{~S}_{-} \mathrm{SO}_{4}$ labeled proteoglycans can be assessed by SDS-PAGE (4-20 \% acrylamide gradient gel) using a fixed amount of radioactivity per lane. The SDS-PAGE gel of the biglycan produced and secreted by vascular smooth muscle cells treated with various concentrations of TGF $\beta$ for $24 \mathrm{~h}$ is shown (Lower Panel) where the decreasing migration of the biglycan band with increasing TGF $\beta$ occurs due to the lengthening of the GAG chains (see text for references). In the context of the current paper, the band in lane 2 (second from left) is natural or basal biglycan and the bands in lanes 3 to 9 are hyperelongated biglycan derived from cells treated with TGF $\beta$ or PDGF (platelet-derived growth factor). See text and references therein for further details. 
lymerization and thus the sequential alternate transfer of GalNAc and GlcA forms the disaccharide repeat units of the polymerizing GAG chain. The GAG chain is subject to sulfation and epimerization to yield further structural heterogeneity [18]. Whereas the core protein has a defined molecular weight derived from its mRNA coding and amino acid structure, the GAG chains have a range of sizes conferring a range of molecular weights to the whole proteoglycan. Approximately 15 enzymes have been cloned which undertake these steps of linkage region and CS GAG synthesis but the manner in which they affect GAG polymerization is unknown [18].

A further important consideration of GAG chain structure in tissues is that numerous factors can stimulate cells to produce modified, particularly elongated GAG chains, which we term hyperelongated GAGs $[10,16]$. Although the outcome of cell stimulation leading to modification of CS GAGs is clearly established, only in the last decade have the signaling pathways begun to be defined and nothing is known of the link between the signaling pathways and the precise biochemical modifications that lead to the changes in GAG structure.

\section{ENZYMES REGULATING THE SYNTHESIS OF CS GAG CHAINS}

GAG chain initiation and attachment to the core protein occurs principally within the linked compartments of the endoplasmic reticulum (ER) and the cis and trans Golgi. CS, DS and heparan sulfate (HS) GAG chains are attached to serine residues of core proteins via an O-linked glycosidic bond. A single consensus sequence for GAG chain initiation has not been defined although serine residues flanked by glycine appear to be involved [18]. A tetra-saccharide linkage region is initiated by the action of the enzyme UDP-Xyl transferase to attach the pentose sugar, Xyl, to a serine residue on the core protein [20]. Addition of the linker region occurs in a compartment intermediate to the endoplasmic reticulum and the Golgi apparatus [1]. Construction of the tetrasaccharide linkage region continues with the addition of two galactose (Gal) units by UDP-Gal transferase I and II respectively and is completed upon addition of GlcA by UDP-GlcA transferase I [21]. The addition of characteristic repeating disaccharide units differs at this point which is a divergence point for the biosynthesis of different types of GAG chains. Intriguingly, modifications including sulfation and phosphorylation of the tetrasaccharide linkage region may influence the resultant GAG types [19, 22, 23].

Multiple enzymes involved in the sequential addition of monosaccharides, have been identified, cloned and their transferase and polymerase activities characterized [24-29]. GalNAc transferase I and II and GlcA transferase II are involved in CS biosynthesis and the enzyme C-5 GlcA epimerase converts CS to DS. For comparison, for heparin and HS synthesis the enzymes EXTL2, EXT1, EXT2, several NDSTs and C-5 GlcA epimerase have been identified [21]. Sulfation of the GAG chains also occurs in the Golgi apparatus, where sulfotransferases add sulfate groups from 3'phosphoadenosine-5' phosphosulfate (PAPS) to specific positions on the emerging polysaccharide chain [30].

\section{TRANSFERASES, POLYMERASES AND THE DE- TERMINATION OF "NATURAL" GAG LENGTH}

The "natural" length of GAG chains is defined as the length of GAG chain synthesized by the GAG elongation machinery operating in a cell which is not stimulated by external stimuli which cause additional elongation of GAG chain length [16] (see Fig. (1) for schematic representation). Presently six homologous enzymes have been cloned which can undertake the addition of a monosaccharide to an acceptor oligosaccharide and thus have the potential to undertake CS GAG polymerization. The designations of the enzymes involved in GAG polymerization have been evolving over the last decade so it is noted that the text of this manuscript utilizes the designations as used in the quoted references and

Table 1. Designation of the six human enzymes associated with chondroitin sulfate glycosaminoglycan chain polymerization. Note that an additional eight or more enzymes can be involved in the synthesis of GAG chains, some of which may impact of GAG elongation, but this table and paper focuses on those enzymes primarily involved in GAG chain polymerization

\begin{tabular}{|c|c|c|c|c|}
\hline Transferase/Polymerase & Name $^{1}$ & EC Number ${ }^{2}$ & Gene Name & References \\
\hline $\begin{array}{c}\text { Chondroitin sulfate } \\
\text { N-acetylgalactosaminyltransferase } 1\end{array}$ & CGAT1 & 2.4.1.174 & CSGALNACT1 & {$[26,27]$} \\
\hline $\begin{array}{c}\text { Chondroitin sulfate } \\
\text { N-acetylgalactosaminyltransferase } 2\end{array}$ & CGAT2 & 2.4.1.174 & CSGALNACT2 & {$[53,28]$} \\
\hline Chondroitin sulfate synthase 1 & CHSS1 & $\begin{array}{l}2.4 .1 .226 \\
2.4 .1 .175\end{array}$ & CHSY1 & {$[24,29]$} \\
\hline Chondroitin sulfate synthase 2 & CHSS2 & $\begin{array}{l}2.4 .1 .226 \\
2.4 .1 .175\end{array}$ & CHPF & [29] \\
\hline
\end{tabular}

${ }^{1}$ Nomenclature of the UniProt Knowledgebase of ExPASy Proteomics server.

${ }^{2}$ Transferase activities are: EC 2.4.1.174, EC 2.4.1.175 is N-acetylgalactosaminyltransferase (GalNAc transferase) activity; EC 2.4.1.226 is Glucuronyltransferase (GlcA transferase) activity. 
these can be checked against the designations compiled in Table 1. Notably, although these enzymes act as monosaccharide transferases, when expressed individually they do not manifest polymerase activity sufficient to generate GAG chains similar to those produced by intact cells. Recent advances described below, have revealed that the enzymes need to be expressed in combinations to achieve polymerization activity. The genes for these enzymes are localized on different chromosomes despite significant homology in the nucleotide and amino acid sequences [31].

None of the individual enzymes possesses polymerase activity in vitro sufficient to produce a run of disaccharide repeat units that would constitute a GAG chain. ChSy-1 and ChPF show similar tissue distributions whereas ChSy-2 and ChSy-3 each show a specific tissue distribution which is distinct from the first mentioned pair of enzymes [32]. ChSy1,2 and 3 along with ChPF have all been shown to colocalise in the Golgi where they work in an orchestrated manner to manifest GAG polymerization [33]. It has emerged that some of these enzymes exhibit transferase and polymerase activity whereas some act as chaperones which bind to partners to generate complexes which show polymerization activity. Importantly, polymerization activity of the different combinations of glycosyltransferases and chaperones is variable and so is the length of the resultant GAG chains which are synthesized by various different combinations of enzymes [32].

The chondroitin glycosyltransferases, $\mathrm{ChGn}-1$ and ChGn-2 (GalNAcT-1 and -2), are apparently not essential for CS GAG polymerization and notably homologues of these transferases are not present in Caenorhabditis elegans which does possess chondroitin GAGs [34]. It is unclear why so many CS synthesizing enzymes exist in nature. It is notable that no cell lines with greatly disrupted CS biosynthesis have been reported and this may be due to a high level of functional redundancy that is conferred by having a family of polymerization enzymes available. The existence of multiple polymerizing enzymes, which when expressed in different relative abundances synthesize GAG chains of different lengths provides an explanation for the existence of variability in the length of GAG chains occurring in different tissues, at different developmental stages and in disease states $[8,35,36]$. Differential expression associated with varying GAG polymerizing activity also provides a possible mechanism for the action of external stimuli to modify GAG length which could occur, for example, by alterations in the relative expression of the enzymes and chaperones. The implications for different GAG lengths in different tissues and in different physiological and pathological states is presently unknown.

Most glycosyltransferases contain a DXD motif which is critical for catalytic function possibly by modulation of the binding of UDP-sugars through the coordination of a divalent cation $[37,38]$. ChPF lacks the usual DXD motif characteristic of Golgi resident enzymes which opens the possibility of a different role from the integral membrane enzymes including the possibility that it may be rate limiting and amenable to altered expression which can alter the length of GAG chains [29].

Kitagawa and colleagues [32] showed that ChPF when expressed alone does not show polymerization activity but when it is expressed with other enzymes it is capable of me- diating chain polymerization. Furthermore, the size of the GAG chains is dependent upon the glycosyltransferase partner. For example, when ChPF is co-expressed and partners with $\mathrm{ChSy}-1$ the resultant GAG chains are longer than when it is coexpresssed with $\mathrm{ChSy}-2$ [32].

Each of ChSy-1,2 and 3 contain one domain with glycosyltransferase activity and accordingly pairs of enzymes carry two functional domains. Kitagawa and colleagues also examined the effect of mutating out the glycosyltransferase activity of ChSy-3 whilst demonstrating that the resultant protein did interact with other glycosyltransferases [33]. When evaluated for polymerization activity it was found that in the ChSy-3:ChSy-1 complex both domains contributed equally to polymerization however for the ChSy2:ChSy-3 complex the ChSy-3 contributed totally to the polymerization activity and the ChSy-2 served only, but critically, as a chaperone [33].

CS polymerization complexes also bind to the proteoglycan core protein or an equivalent initiation protein and the nature of the core protein can determine the extent of GAG polymerization observed. Furthermore, CS GAG synthesis is initiated on xyloside derivatives $[3,39]$ and the nature of the hydrophobic aglycone of the xyloside derivative can determine both the type of GAG chain as well as the length of the resultant chains. Thus, the polymerizing activity of the various transferase complexes is modulated by the interaction with the recognition sequence of the proteoglycan core protein or artificially by the nature of the hydrophobic aglycone of a xyloside derivative.

Enzymatic sulfation during synthesis of the GAG chain is suggested to play a role in determining ultimate GAG length. Sulfation proceeds with polymerization and specific sulfate groups have stimulatory or inhibitory effects on GalNAc and GlcA transfer [24, 40]. Early studies suggested that 4,6-O sulfation on GalNAc and 3-O-sulfation on GlcA residues at the non-reducing end might be involved in terminating polymerization of CS GAG chains [41]. A deeper understanding of the processes of CS GAG polymerization will almost certainly provide insights into the molecular mechanisms through which supplementary chain modifications such as sulfation modulate GAG length and may therefore reveal tissue specific effects which will inform considerations of function.

Protein:protein interactions are $\mathrm{pH}$ sensitive and the $\mathrm{pH}$ alters throughout the Golgi apparatus. The $\mathrm{pH}$ generally decreases along the secretory pathway within the Golgi with a variation of as much as $0.5 \mathrm{pH}$ units [33]. Coexpression of pairs of ChSy-3, ChSy-1 and ChPF using GlcUA $31-3 \mathrm{Gal} \beta \mathrm{O}-\mathrm{NM}$ as an acceptor generates GAGs of differing length and the length is dependent upon the $\mathrm{pH}$ of the assay [33]. Thus, the localization of the glycosyltransferases in the Golgi and the $\mathrm{pH}$ at the relevant position becomes another determinant of natural GAG length.

Thus, full polymerization activity requires a complex of at least two of the four critical polymerization enzymes (ChSy-1, -2, -3 and $\mathrm{ChPF}$ ) and the resultant polymerization capacity is variable depending upon the entities that form the complexes and the core protein to which they direct their activity. In summary, it is possible to speculate that the factors that determine natural GAG length in tissues are the 
specific and relative level of expression of the six or perhaps only four enzymes shown in Table 1 (i.e. requirement for transferase versus polymerase activity), the respective core protein, the $\mathrm{pH}$ of the Golgi subcompartments and the sulfation of the disaccharides in the forming GAG chain.

\section{MECHANISMS LEADING TO THE HYPERELON- GATION OF CS GAG CHAINS}

Cells synthesize CS proteoglycans with GAG chains of "natural" length due to the basal activity of the enzymes, transporters and processes associated with GAG biosynthesis in the cell, as described above. However, treatment of cells such as vascular smooth muscle cells (VSMC) with growth factors such as platelet-derived growth factor (PDGF), transforming growth factor (TGF) $\beta$, angiotensin II and metabolic agents including oxLDL leads to the synthesis and secretion of CS/DS proteoglycans which are larger than those from untreated cells [3, 12, 42, 43]. For a specific example of the effect of treating cells with TGF $\beta$ on the size of the resultant proteoglycans see Fig. (1) (lower panel). Size increases of proteoglycans are due to elongation of the GAG chains which can be demonstrated by the chemical cleavage of the chains from the core proteins and GAG size analysis by SDS-PAGE, size exclusion chromatography or fluorophore assisted carbohydrate electrophoresis (FACE) [3, 44]. The increase in size of the GAG chains is approximately 10-20 per cent [3]. Some growth factors such as PDGF also cause an increase in the 6-sulfation of the CS/DS GAG chain but others such as TGF $\beta$ only cause GAG elongation [45]. CS GAG chains may also be modified by epimerization which changes the sugars from GlcA to IdoA (and hence CS to DS GAG chains). Although this has the potential to modify the interaction with apolipoproteins little is known of the role of growth factors and other stimuli in regulating this reaction [18]. We have observed that GAGs secreted by cultured VSMCs have a particularly high CS versus DS content suggesting that epimerase activity may be down regulated in such cultured cells (Little and Nigro, unpublished observation).

The signaling pathways downstream of receptor activation mediating GAG hyperelongation are complex and in some cases novel pathways appear to be involved. Using the example of VSMCs several growth factors including PDGF, TGF $\beta$ and thrombin have been shown to mediate GAG (hyper)elongation and the signaling pathways eliciting this response have been partially characterized. Schonherr et al. [5] reported that PDGF stimulates GAG elongation of CS GAGs by monkey VSMCs however the response is not blocked by the isoflavone, genistein. Genistein, the prototypical inhibitor of the intrinsic tyrosine kinase autotransphosphorylation activity of the dimeric PDGF receptor and is an inhibitor of PDGF-mediated mitogenic signaling in VSMC [5]. The data of Schonherr et al. [5] leads to the implication that there may be a PDGFR tyrosine kinase independent signal transduction pathway controlling GAG elongation. There is evidence for this in that PDGF-stimulated early growth response gene (egr)-1 expression in $3 \mathrm{~T} 3$ fibroblasts cells is also not blocked by genistein [46].

The action of TGF $\beta$ to stimulate CS/DS GAG elongation in human VSMCs involves the canonical Smad/phosphoSmad 2/3 pathway but also has the novel aspect that it en- gages the p38/phospho p38 Mitogen Activated Protein Kinase (MAPK) pathway usually associated with stress signaling [13].

The action of the serine protease, thrombin to stimulate GAG elongation in VSMCs is mediated via the Protease Activated Receptor-1 and involves protein kinase $\mathrm{C}$ and transactivation of the Epidermal Growth Factor receptor [47].

The precise biochemical mechanism(s) through which the signaling pathways activated by growth factors lead to hyperelongation of GAGs is unknown. ALK V/Smad and p38 MAPK signaling pathways generate signaling intermediates which move to the nucleus and act as transcription factors. These transcription factors may for example alter the expression of the GAG polymerizing enzymes however studies of the regulation of GAG polymerizing enzymes have so far not yielded substantial advances [13, 48]. ChPF has recently been discovered and shown to play a role in CS GAG polymerization in vitro [32]. Whether or not the growth factors may be regulating the expression and action of $\mathrm{ChPF}$ is an interesting question. ChSy-1 leads to longer GAG chains in vitro than $\mathrm{ChSy}-2$ (when each is co expressed with $\mathrm{ChPF}$ ) so one possibility is that hyperelongation may occur due to a shift in the major enzyme from ChSy-2 to ChSy-1. For example, both enzymes may be involved in the synthesis of natural length GAG chains - leading to the spectrum of chain sizes observed - and growth factors may stimulate the expression of ChPF which is targeted within the cell at ChSy-1 thus yielding longer GAG chains.

\section{DIFFERENTIATING NATURAL GAG SYNTHESIS FROM GAG HYPERELONGATION}

GAG formation is performed by a number of membrane bound enzymes located in the Golgi apparatus [18]. As discussed, a variety of agonists can stimulate elongation of GAG chains [3, 12, 42, 43]. The signaling pathways through which agonists mediate GAG elongation are being revealed and represent known and possibly novel pathways. It is unknown how these signaling pathways mediate their effects to cause GAG elongation. An interesting question is whether or not natural GAG synthesis and agonist-mediated hyperelongation involves the synthesis of new proteins? Vijayagopol and colleagues [49] showed that a growth factor mixture (conditioned media) derived from endothelial cells and containing TGF $\beta$ stimulated an increase in the size of proteoglycans and thus by implication the size of the GAG chains produced by bovine aortic smooth muscle cells. The GAG elongation was blocked by cycloheximide which prevents de novo protein synthesis and also by the transcription inhibitor actinomycin D [49]. We have recently confirmed some of these results in human VSMCs where cycloheximide inhibits PDGF mediated GAG elongation (Ballinger and Little, unpublished observations). The data that cycloheximide inhibits growth factor mediated GAG elongation but not basal GAG synthesis, demonstrates that the synthesis of natural GAGs does not involve de novo protein synthesis (in the relatively short timeframe of the experiment) but the ability of growth factors to cause GAG hyperelongation is dependent upon de novo protein synthesis. There are several possible interpretations of these results. It is likely that the integral membrane enzymes mediating the synthesis of natu- 
ral GAGs have a slow turnover rate due to the more stable environment within the membranes of the Golgi apparatus. The Golgi resident enzyme galactosyltransferase in which the mature enzyme is phosphorylated has an average half life of about 19 hours $[50,51]$. Although it is obvious that there must be some turnover and thus new synthesis of enzymes required for GAG synthesis it is possible that the need to replenish these enzymes as they are naturally degraded is very slow. Although the synthesis of natural GAGs on proteoglycans does not require de novo protein synthesis the action of growth factors to hyperelongate GAGs is clearly dependent upon the synthesis of new protein. There are at least two possibilities for the role of this newly synthesized protein in mediating GAG elongation - either the signaling pathway linking receptor activation and GAG hyperelongation involves gene expression and new protein synthesis or the agonist is causing the increased expression and synthesis of a factor or cofactor which is rate limiting in GAG synthesis [32]. For example, in the pathway that TGF $\beta$ stimulates biglycan expression in PANC-1 and MG-63 cells, the initial stimulation of Smad phosphorylation is followed by the expression and synthesis of GADD45 $\beta$ which activates p38 MAPK [52]. This is a rapid response and it would be expected that acute cycloheximide treatment could block this pathway.

Thus, there appears to be a clear difference between natural GAG synthesis and GAG hyperelongation and a schema is shown in Fig. (2). The fact that GAG hyperelongation has very specific signaling pathways that involve de novo protein synthesis further validates GAG hyperelongation as a potential therapeutic target $[14,16,17]$.

\section{CONCLUSIONS}

Recent advances in the cloning and expression of CS glycosyltransferases have identified six functional enzymes, four of which appear to be the most important for CS GAG polymerization. These proteins work in combination, certainly at least in pairs, to manifest CS GAG polymerization to form tissue specific "natural" CS GAG polymers. The relative level of expression of the respective enzymes, the specific proteoglycan core protein, $\mathrm{pH}$ of the Golgi subcompartments and the activity of sulfotransferases are factors that determine natural GAG length.

Superimposed on this basic cell biology of CS GAG synthesis are the external factors that have classic cell surface receptors and intracellular signaling pathways that can modify the ultimate GAG length most specifically leading to GAG elongation of the order of 20 percent. This GAG elongation is functionally significant because it leads to enhanced binding to LDL which is closely associated with the earliest stages of the initiation of atherosclerosis [9, 14]. How the signaling pathways link to GAG elongation and whether or not this is due to an impact on natural GAG synthesis or the superimposition of a distinct process is presently unknown. This is an important question because if GAG hyperelongation can be separated from natural GAG synthesis then it

\section{Proteoglycan synthesis}

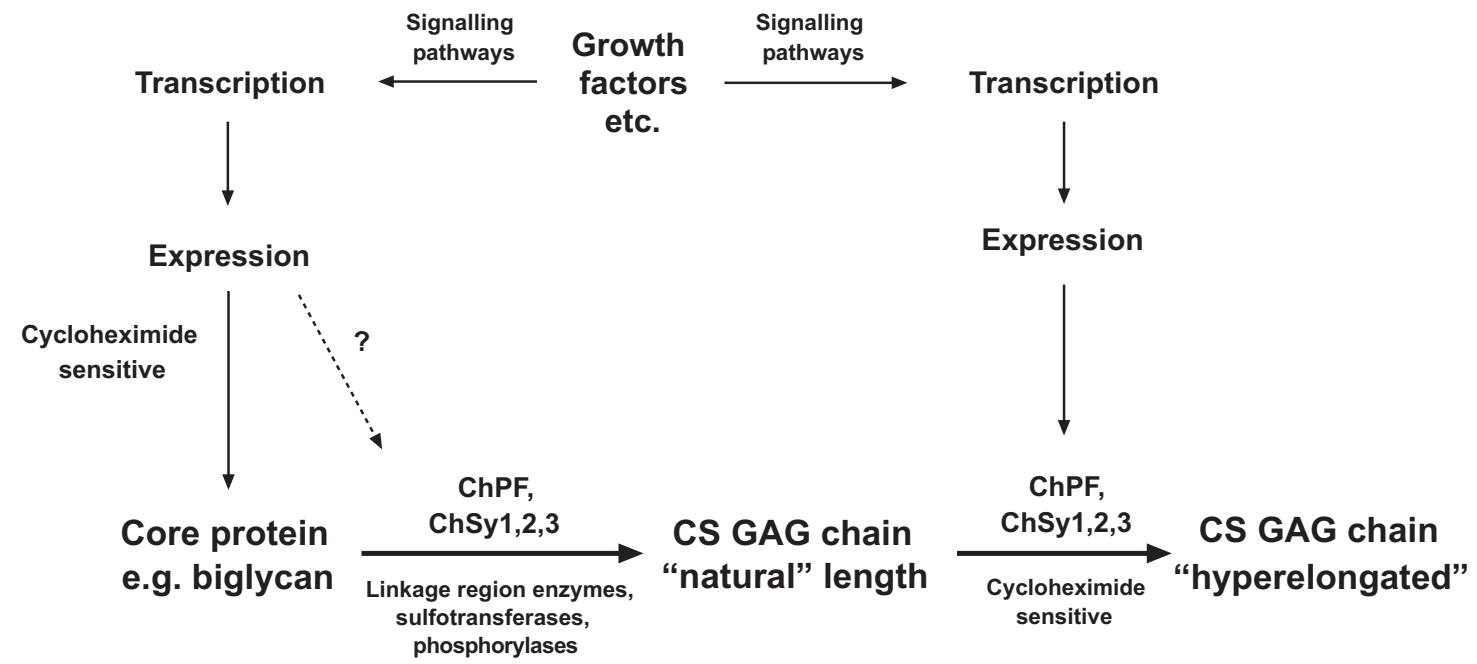

Fig. (2). Schema of the biochemical processes leading to the synthesis of natural chondroitin sulfate glycosaminoglycan (GAG) chains and the hyperelongation of GAG chains elicited by multiple cell signaling pathways resulting from the action of growth factor such as PDGF and TGF $\beta$. ChSy chondroitin synthase; ChPF chondroitin polymerizing factor. The scheme to the left shows the normal biosynthesis of a proteoglycan core protein which is new protein and therefore cycloheximide sensitive but is also subject, based on individual and specific core proteins, to up regulation by growth factors. Various combinations, at least pairs of polymerases (see Table 1) add monosaccharides to the preformed tetrasaccharide linkage region on a serine residue on the core protein to produce the "natural" length glycosaminoglycan (GAG) chain. To the right of the scheme it is indicated that growth factors can intervene to result in the production of longer, so-called hyperelongated GAG chains and although this is cycloheximide sensitive (see text) and acutely requires new protein synthesis the site at which the new protein synthesis occurs, either in the signaling pathway or in the actual transporters and enzymes that synthesis GAGs is unknown. The GAG elongation in response to growth factors is of the order of 20 per cent but this is sufficient to half the affinity of binding of the GAG chain to LDL. 
may lead to identification of a therapeutic target which allows for the prevention of disease without interfering with the physiological role of proteoglycans.

\section{ACKNOWLEDGEMENTS}

The experimental work underpinning this review has been supported by research grants from the National Health and Medical Research Council of Australia Project Grants ("268928 and \#472611) and Development Grant ( $\left.{ }^{\#} 418934\right)$ (PJL), project grants from the Diabetes Australia Research Trust (PJL) (NO) and a Grant-in-Aid from the National Heart Foundation of Australia (PJL).

\section{CONFLICTS OF INTEREST}

PJL and BakerIDI Heart and Diabetes Institute hold a provisional patent application for a set of compounds that inhibit GAG hyperelongation and may have a use in diseases associated with that condition. Nothing else to declare.

\section{REFERENCES}

[1] Hascall, V. C.; Heinegard, D.K.; Wight, T. N. In Cell Biology of Extracellular Matrix, Hay, E. D., Ed., Plenum Press, New York 1991; pp. 149-175.

[2] Wight, T. N. In Atherosclerosis, Gotlieb, A. I., Ed., Plenum Press, New York 1991; pp. 115-125.

[3] Little, P. J.; Tannock, L.; Olin, K. L.; Chait, A.; Wight, T.N. Proteoglycans synthesized by arterial smooth muscle cells in the presence of transforming growth factor-betal exhibit increased binding to LDLs. Arterioscler. Thromb. Vasc. Biol., 2002, 22, 55-60.

[4] Linhardt, R. J.; Toida, T. Role of glycosaminoglycans in cellular communication. Acc. Chem. Res., 2004, 37, 431-8.

[5] Schonherr, E.; Kinsella, M.G.; Wight, T. N. Genistein selectively inhibits platelet-derived growth factor- stimulated versican biosynthesis in monkey arterial smooth muscle cells. Arch. Biochem. Biophys., 1997, 339, 353-61.

[6] Silbert, C.K.; Palmer, M.E.; Humphries, D.E.; Silbert, J.E. Production of $[3 \mathrm{H}]$ hexosamine-labeled proteoglycans by cultures of normal and diabetic skin fibroblasts: dilution of exogenous $[3 \mathrm{H}]$ glucosamine by endogenous hexosamine from glucose and other sources. Arch. Biochem. Biophys., 1989, 268, 393-7.

[7] O'Brien, K.D.; Olin, K.L.; Alpers, C.E.; Chiu, W.; Ferguson, M.; Hudkins, K.; Wight, T.N.; Chait, A. Comparison of apolipoprotein and proteoglycan deposits in human coronary atherosclerotic plaques: colocalization of biglycan with apolipoproteins. Circulation, 1998, 98, 519-27.

[8] Plaas, A.H.; West, L.A.; Wong-Palms, S.; Nelson, F.R. Glycosaminoglycan sulfation in human osteoarthritis. Disease-related alterations at the non-reducing termini of chondroitin and dermatan sulfate. J. Biol. Chem., 1998, 273, 12642-9.

[9] Nakashima, Y.; Fujii, H.; Sumiyoshi, S.; Wight, T.N.; Sueishi, K. Early human atherosclerosis: accumulation of lipid and proteoglycans in intimal thickenings followed by macrophage infiltration. Arterioscler. Thromb. Vasc. Biol., 2007, 27, 1159-65.

[10] Little, P.J.; Osman, N.; O'Brien, K.D. Hyperelongated biglycan: the surreptitious initiator of atherosclerosis. Curr. Opin. Lipidol., 2008, 19, 448-454.

[11] Nigro, J.; Ballinger, M.; Dilley, R.; Jennings, G.; Wight, T.; Little, P. Fenofibrate modifies human vascular smooth muscle proteoglycans and reduces LDL binding. Diabetologia, 2004, 47, 2105-13.

[12] Schonherr, E.; Jarvelainen, H.T.; Kinsella, M.G.; Sandell, L.J.; Wight, T.N. Platelet-derived growth factor and transforming growth factor-beta 1 differentially affect the synthesis of biglycan and decorin by monkey arterial smooth muscle cells. Arterioscler. Thromb., 1993, 13, 1026-36.

[13] Dadlani, H.; Ballinger, M.L.; Osman, N.; Getachew, R.; Little, P.J. Smad and p38 MAP kinase-mediated signaling of proteoglycan synthesis in vascular smooth muscle. J. Biol. Chem., 2008, 283, 7844-52.

[14] Little, P. J.; Ballinger, M.L.; Osman, N. Vascular wall proteoglycan synthesis and structure as a target for the prevention of atherosclerosis. Vasc. Health Risk Manag., 2007, 3, 1-8.
[15] Camejo, G. The interaction of lipids and lipoproteins with the intercellular matrix of arterial tissue: its possible role in atherogenesis. Adv. Lipid Res., 1982, 19, 1-53.

[16] Ballinger, M.L.; Nigro, J.; Frontanilla, K.V.; Dart, A.M.; Little, P. J. Regulation of glycosaminoglycan structure and atherogenesis. Cell Mol. Life Sci., 2004, 61, 1296-306.

[17] Grande-Allen, K.J.; Osman, N.; Ballinger, M.L.; Dadlani, H.; Marasco, S.; Little, P. J. Glycosaminoglycan synthesis and structure as targets for the prevention of calcific aortic valve disease. Cardiovasc. Res., 2007, 76, 19-28.

[18] Silbert, J.E.; Sugumaran, G. Biosynthesis of chondroitin/dermatan sulfate. IUBMB Life, 2002, 54, 177-86.

[19] Tone, Y.; Pedersen, L.C.; Yamamoto, T.; Izumikawa, T.; Kitagawa, H.; Nishihara, J.; Tamura, J.; Negishi, M.; Sugahara, K. 2-ophosphorylation of xylose and 6-o-sulfation of galactose in the protein linkage region of glycosaminoglycans influence the glucuronyltransferase-I activity involved in the linkage region synthesis. J. Biol. Chem., 2008, 283, 16801-7.

[20] Schwartz, N. Biosynthesis and regulation of expression of proteoglycans. Front Biosci., 2000, 5, D649-55.

[21] Prydz, K.Dalen, K. T. Synthesis and sorting of proteoglycans. $J$. Cell Sci., 2000, 113 (Pt 2), 193-205.

[22] Gulberti, S.; Lattard, V.; Fondeur, M.; Jacquinet, J.C.; Mulliert, G. Netter, P.; Magdalou, J.; Ouzzine, M.; Fournel-Gigleux, S. Modifications of the glycosaminoglycan-linkage region of proteoglycans: phosphorylation and sulfation determine the activity of the human beta1,4-galactosyltransferase 7 and beta1,3-glucuronosyltransferase I. Sci. World J., 2005, 5, 510-4.

[23] Gulberti, S.; Lattard, V.; Fondeur, M.; Jacquinet, J.C.; Mulliert, G.; Netter, P.; Magdalou, J.; Ouzzine, M.; Fournel-Gigleux, S. Phosphorylation and sulfation of oligosaccharide substrates critically influence the activity of human beta1,4-galactosyltransferase 7 (GalT-I) and beta1,3-glucuronosyltransferase I (GlcAT-I) involved in the biosynthesis of the glycosaminoglycan-protein linkage region of proteoglycans. J. Biol. Chem., 2005, 280, 1417-25.

[24] Kitagawa, H.; Uyama, T.; Sugahara, K. Molecular cloning and expression of a human chondroitin synthase. J. Biol. Chem., 2001, 276, 38721-6.

[25] Yada, T.; Sato, T.; Kaseyama, H.; Gotoh, M.; Iwasaki, H.; Kikuchi, N.; Kwon, Y. D.; Togayachi, A.; Kudo, T.; Watanabe, H.; Narimatsu, H.; Kimata, K. Chondroitin sulfate synthase-3. Molecular cloning and characterization. J. Biol. Chem., 2003, 278, 39711-25.

[26] Gotoh, M.; Yada, T.; Sato, T.; Akashima, T.; Iwasaki, H.; Mochizuki, H.; Inaba, N.; Togayachi, A.; Kudo, T.; Watanabe, H.; Kimata, K.; Narimatsu, H. Molecular cloning and characterization of a novel chondroitin sulfate glucuronyltransferase that transfers glucuronic acid to N-acetylgalactosamine. J. Biol. Chem., 2002, 277, 38179-88.

[27] Uyama, T.; Kitagawa, H.; Tamura, Ji, J.; Sugahara, K. Molecular cloning and expression of human chondroitin $\mathrm{N}$ acetylgalactosaminyltransferase: the key enzyme for chain initiation and elongation of chondroitin/dermatan sulfate on the protein linkage region tetrasaccharide shared by heparin/heparan sulfate. $J$. Biol. Chem., 2002, 277, 8841-6.

[28] Uyama, T.; Kitagawa, H.; Tanaka, J.; Tamura, J.; Ogawa, T.; Sugahara, K. Molecular cloning and expression of a second chondroitin $\mathrm{N}$-acetylgalactosaminyltransferase involved in the initiation and elongation of chondroitin/dermatan sulfate. J. Biol. Chem., 2003, 278, 3072-8.

[29] Kitagawa, H.; Izumikawa, T.; Uyama, T.; Sugahara, K. Molecular cloning of a chondroitin polymerizing factor that cooperates with chondroitin synthase for chondroitin polymerization. J. Biol. Chem., 2003, 278, 23666-71.

[30] Kusche-Gullberg, M.; Kjellen, L. Sulfotransferases in glycosaminoglycan biosynthesis. Curr. Opin. Struct. Biol., 2003, 13, 605-11.

[31] Yada, T.; Gotoh, M.; Sato, T.; Shionyu, M.; Go, M.; Kaseyama, H.; Iwasaki, H.; Kikuchi, N.; Kwon, Y.D.; Togayachi, A.; Kudo, T.; Watanabe, H.; Narimatsu, H.; Kimata, K. Chondroitin sulfate synthase-2. Molecular cloning and characterization of a novel human glycosyltransferase homologous to chondroitin sulfate glucuronyltransferase, which has dual enzymatic activities. J. Biol. Chem., 2003, 278, 30235-47.

[32] Izumikawa, T.; Uyama, T.; Okuura, Y.; Sugahara, K.; Kitagawa, H. Involvement of chondroitin sulfate synthase-3 (chondroitin synthase-2) in chondroitin polymerization through its interaction with 
chondroitin synthase-1 or chondroitin-polymerizing factor. Biochem. J., 2007, 403, 545-52.

[33] Izumikawa, T.; Koike, T.; Shiozawa, S.; Sugahara, K.; Tamura, J.; Kitagawa, H. Identification of Chondroitin Sulfate Glucuronyltransferase as Chondroitin Synthase-3 Involved in Chondroitin Polymerization: Chondroitin polymerization is achieved by multiple enzyme complexes consisting of chondroitin synthase family members. J. Biol. Chem., 2008, 283, 11396-406.

[34] Yamada, S.; Van Die, I.; Van den Eijnden, D.H.; Yokota, A.; Kitagawa, H.; Sugahara, K. Demonstration of glycosaminoglycans in Caenorhabditis elegans. FEBS Lett., 1999, 459, 327-31.

[35] Lauder, R.M.; Huckerby, T.N.; Nieduszynski, I.A.; Plaas, A.H. Age-related changes in the structure of the keratan sulphate chains attached to fibromodulin isolated from articular cartilage. Biochem. J., 1998, 330 ( Pt 2), 753-7.

[36] Sharma, A.; Wood, L.D.; Richardson, J.B.; Roberts, S.; Kuiper, N.J. Glycosaminoglycan profiles of repair tissue formed following autologous chondrocyte implantation differ from control cartilage. Arthritis. Res. Ther., 2007, 9, R79.

[37] Boeggeman, E.; Qasba, P.K. Studies on the metal binding sites in the catalytic domain of beta1,4-galactosyltransferase. Glycobiology, 2002, 12, 395-407.

[38] Pedersen, L.C.; Tsuchida, K.; Kitagawa, H.; Sugahara, K.; Darden, T.A.; Negishi, M. Heparan/chondroitin sulfate biosynthesis. Structure and mechanism of human glucuronyltransferase I. J. Biol. Chem., 2000, 275, 34580-5.

[39] Fritz, T.A.; Esko, J. D. In Proteoglycan Protocols, Iozzo, R. V., Ed., Humana Press Inc, Totowa, NJ 2001; pp. 317-324.

[40] Gundlach, M.W.; Conrad, H.E. Glycosyl transferases in chondroitin sulphate biosynthesis. Effect of acceptor structure on activity. Biochem. J., 1985, 226, 705-14.

[41] Kitagawa, H.; Tsutsumi, K.; Ujikawa, M.; Goto, F.; Tamura, J.; Neumann, K. W.;Ogawa, T.; Sugahara, K. Regulation of chondroitin sulfate biosynthesis by specific sulfation: acceptor specificity of serum beta-GalNAc transferase revealed by structurally defined oligosaccharides. Glycobiology, 1997, 7, 531-7.

[42] Figueroa, J.E.; Vijayagopal, P. Angiotensin II stimulates synthesis of vascular smooth muscle cell proteoglycans with enhanced low density lipoprotein binding properties. Atherosclerosis, 2002, 162, 261-268.

[43] Chang, M.Y.; Potter-Perigo, S.; Tsoi, C.; Chait, A.; Wight, T.N. Oxidized low density lipoproteins regulate synthesis of monkey aortic smooth muscle cell proteoglycans that have enhanced native low density lipoprotein binding properties. J. Biol. Chem., 2000, 275, 4766-73.

[44] Calabro, A.; Hascall, V.C.; Midura, R.J. Adaptation of FACE methodology for microanalysis of total hyaluronan and chondroitin sulfate composition from cartilage. Glycobiology, 2000, 10, 28393.

[45] Schonherr, E.; Jarvelainen, H.T.; Sandell, L.J.; Wight, T.N. Effects of platelet-derived growth factor and transforming growth factorbeta 1 on the synthesis of a large versican-like chondroitin sulfate proteoglycan by arterial smooth muscle cells. J. Biol. Chem., 1991, 266, 17640-7.

[46] Mundschau, L.J.; Forman, L.W.; Weng, H.; Faller, D.V. Plateletderived growth factor (PDGF) induction of egr-1 is independent of PDGF receptor autophosphorylation on tyrosine. J. Biol. Chem., 1994, 269, 16137-42.

[47] Ivey, M.E.; Little, P.J. Thrombin regulates vascular smooth muscle cell proteoglycan synthesis via PAR-1 and multiple downstream signalling pathways. Thromb. Res., 2008; Jun 19 [Epub ahead of print].

[48] Massague, J.; Seoane, J.; Wotton, D. Smad transcription factors. Genes Dev., 2005, 19, 2783-810.

[49] Ciolino, H.P.; Vijayagopal, P.; Berenson, G.S. Endothelial cellconditioned medium modulates the synthesis and structure of proteoglycans in vascular smooth muscle cells. Biochim. Biophys. Acta, 1992, 1135, 129-40.

[50] Strous, G. J.; Berger, E.G. Biosynthesis, intracellular transport, and release of the Golgi enzyme galactosyltransferase (lactose synthetase A protein) in HeLa cells. J. Biol. Chem., 1982, 257, 7623-8.

[51] Strous, G. J.;van Kerkhof, P.;Fallon, R. J.Schwartz, A. L. Golgi galactosyltransferase contains serine-linked phosphate. Eur. J. Biochem., 1987, 169, 307-11.

[52] Ungefroren, H.;Groth, S.;Ruhnke, M.;Kalthoff, H.Fandrich, F. Transforming growth factor-beta (TGF-beta) type I receptor/ALK5-dependent activation of the GADD45beta gene mediates the induction of biglycan expression by TGF-beta. J. Biol. Chem., 2005, 280, 2644-52.

[53] Sato, T.; Gotoh, M.; Kiyohara, K.; Akashima, T.; Iwasaki, H.; Kameyama, A.; Mochizuki, H.; Yada, T.; Inaba, N.; Togayachi, A.; Kudo, T.; Asada, M.; Watanabe, H.; Imamura, T.; Kimata, K.; Narimatsu, H. Differential roles of two N-acetylgalactosaminyltransferases, CSGalNAcT-1, and a novel enzyme, CSGalNAcT-2. Initiation and elongation in synthesis of chondroitin sulfate. J. Biol. Chem., 2003, 278, 3063-71.

Received: September 01, 2008

Revised: September 10, 2008

Accepted: September 14, 2008

(C) Little et al.; Licensee Bentham Open.

This is an open access article licensed under the terms of the Creative Commons Attribution Non-Commercial License (http://creativecommons.org/licenses/by-nc/3.0/) which permits unrestricted, non-commercial use, distribution and reproduction in any medium, provided the work is properly cited. 